\title{
Study of $1-\mathrm{THz} 4^{\text {th }}$-Harmonic Gyrotron
}

\author{
Chao-Hai Du, Senior Member, IEEE, Fan-Hong Li, Shi Pan, and Pu-Kun Liu, senior \\ Member, IEEE, Liang Zhang, Wen-Long He, Hua-Bi Yin, K. Ronald and Adrian W. Cross
}

\begin{abstract}
A preliminary design of a 1-THz gyrotron based on the fourth harmonic operation is presented. The cylindrical-waveguide $\mathrm{TE}_{4,8}$ mode is selected as the operating mode. Considering the weak beam-wave coupling strength in a $4^{\text {th }}$-harmonic open-cavity interaction circuit at the frequency of $1 \mathrm{THz}$, this paper proposes applying low-temperature cooling, for example applying liquid nitrogen, to increase the limit of the $Q$ factor and extend the effective length of the cavity resulting in a significant enhancement of the interaction efficiency and suppression of ohmic losses. Applying multi-mode time-domain theory in the simulation, it was found that although the competing third-harmonic $\mathrm{TE}_{3,6}$ mode was excited first, the fourth-harmonic $T_{4,8}$ mode finally dominates in the steady state regime.
\end{abstract}

Index Terms-Electron cyclotron maser, gyrotron, ohmic loss, quality factor, sectioned cavity, terahertz.

\section{INTRODUCTION}

Terahertz(THz) waves, occupy a part of the electromagnetic spectrum, which plays an important role in this frequency band giving rise to many potential applications, e.g., high resolution imaging, plasma fusion diagnostics[1] and dynamic nuclear polarization enhanced nuclear magnetic resonance[2]. Traditional vacuum electronic devices operate normally at frequencies lower than the millimeter wave band, while traditional optical devices operate normally at frequencies in the infrared band or higher. As a result, the band between millimeter waves and infrared waves there is a spectral gap known as the "THz gap". To fill this gap scientists with experience in the generation of electromagnetic radiation at microwave frequencies are trying to extend the operating frequency from the microwave band to the $\mathrm{THz}$ band, while those working in optics are devoting resources to lowering the operating frequencies to the $\mathrm{THz}$ band from the infrared sources they have traditionally worked on[3]. However, optical devices such as quantum cascade lasers and pumped terahertz lasers are low power, usually not larger than a milliwatt[3].

This manuscript is submitted on 22nd, March, 2019. This work is sponsored by the National Natural Science Foundation of China under Contracts 61531002, 61861130367 (Newton Advanced Fellowship NAFIR1\180121), and NSAF U1830201. (Corresponding authors: Chao-Hai Du.)

C. H. Du, F. H. Li, S. Pan and P. K. Liu are with the School of Electronics Engineering and Computer Science, Peking University, Beijing 100871, China (e-mail: duchaohai@pku.edu.cn)

L. Zhang, W. L. He, H. B. Yin, A.W. Cross and K. Ronald are with the Department of Physics, SUPA, University of Strathclyde, Glasgow G4 ONG, United Kingdom. W.L. He is also with the College of Electronic Science and Technology, Shenzhen University, Shenzhen 518061, China.
Gyrotrons are a kind of vacuum electric device based on the principle of the electron cyclotron maser [4]. To extend the operating frequency higher than $1 \mathrm{THz}$, some experimental designs have been proposed. A second-harmonic, $1.005-\mathrm{THz}$ gyrotron was confirmed experimentally with a $T E_{6,11}$ cavity operating mode using a 19.0-T pulse magnet at the University of Fukui, Japan [5]. Another experiment designed, manufactured and tested at the Institute of Applied Physics, Russia Academy of Sciences [6]generated $1.5 \mathrm{~kW}$ of coherent radiation at $1.022 \mathrm{THz}$. The power is generated due to the fundamental harmonic interaction, as a result of which, the external magnetic field was as high as $38.5 \mathrm{~T}$. The experiments mentioned above in Japan and Russia both necessitate $>19 \mathrm{~T}$ magnetic fields. To lower the value of the external magnetic field, we propose a design of a 1-THz gyrotron with the fourth-harmonic interaction. The paper is organized as follows. In Section II, low-temperature cooling is introduced to reduce the ohmic loss. In Section III, a sectioned cavity is analyzed based on a single-mode frequency-domain theory. In Section IV, the results of dynamic simulation to analyze the mode competition are presented. Section V summarizes the main conclusions of the work.

\section{LOW TEMPERATURE COOLING}

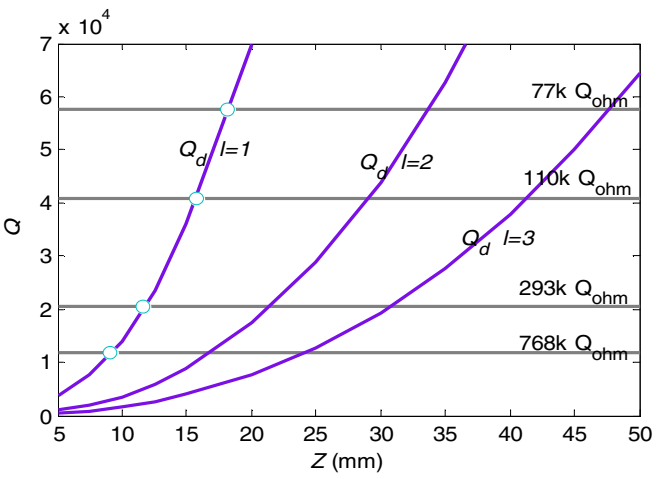

Fig. 1 Diffraction Q-factors under various temperatures. The abscissa is the length of interaction circuit. $l$ is the order of axial modes. The gray solid lines indicate the ohmic Q-factor at different temperatures with the unit in Kelvins.

The output power will decrease rapidly due to the ohmic loss[7]. To diminish the ohmic loss caused by the thermal effect, we propose to lower the temperature of the interaction circuit. The total quality factor (Q-factor) can be determined as follows,

$$
1 / Q=1 / Q_{d}+1 / Q_{o h m}
$$

while $Q$ is the total Q-factor, $Q_{d}$ and $Q_{o h m}$ is diffraction Q-factor and ohmic Q-factor, respectively. $Q_{d}$ at different interaction lengths are calculated based on the single-mode 
frequency-domain theory [8] and are shown in Fig. 1. With the increase of the interaction length, the diffraction Q-factor is increased. However, the total Q-factor is dominated by the Ohmic Q-factor. With the decrease of the circuit temperature as well as the ohmic loss, the upper limit of Q-factor is increased. The start oscillation currents are calculated as demonstrated in Fig. 2. The start oscillation currents of $T E_{3,6}^{(3)}$ and $T E_{4,8}^{(4)}$ modes are lower than the operating current, i.e., 0.7A, thus, further analysis needs to be implemented for evaluating the mode competition. The superscript indicates the harmonic number.

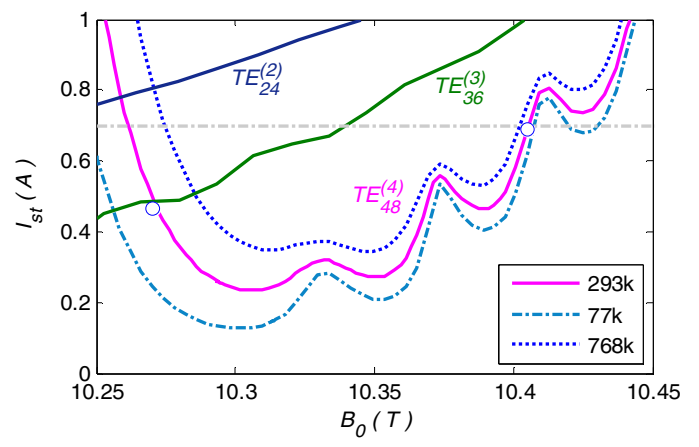

Fig. 2 Start oscillation currents at different temperatures. The gray dash-dotted line is the operating current produced by the electron gun, i.e., 0.7A.

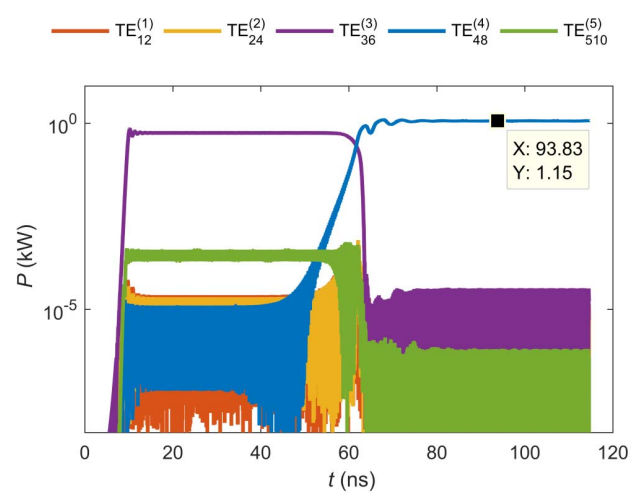

(a)

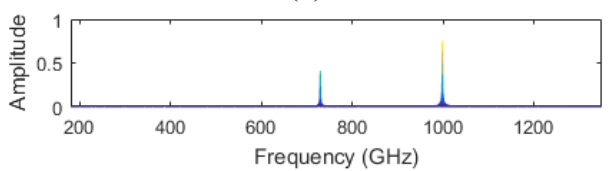

(b)

Fig. 3. (a) The dynamic calculation results and (b) is the frequency spectrum.

\section{DYNAMIC ANALYSIS}

An 80-kV, 0.7-A electron beam is applied to excite the fourth harmonic gyrotron. The pitch factor of the electron gun is 1.5 , and the velocity spread is $10 \%$. The results are verified by the Finite Element Method simulation with the operating mode selected here the $T E_{4,8}^{(4)}$ mode, To analyze the mode competition between the $T E_{4,8}^{(4)}$ mode and adjacent modes, the dynamic simulations were carried out using a multi-mode time-domain program [8]. The results are presented in Fig. . According to the start oscillation currents demonstrated in Fig. 2, the dominant competition modes is between the $T E_{3,6}^{(3)}$ and $T E_{4,8}^{(4)}$ modes. Since the latter has a lower starting oscillation current than the former, the $T E_{3,6}^{(3)}$ can be suppressed and the $T E_{4,8}^{(4)}$ mode prevails existing in the final steady state. The output power of $T E_{4,8}^{(4)}$ mode is five orders of magnitude higher than those of the $T E_{3,6}^{(3)}, T E_{510}^{(5)}, T E_{1,2}^{(1)}$ and $T E_{2,4}^{(2)}$ modes. From the frequency spectrum calculated from the Fourier transform, power around $750 \mathrm{GHz}$ is generated by $T E_{3,6}^{(3)}$ mode and the the $T E_{4,8}^{(4)}$ mode dominates $1 \mathrm{THz}$.

\section{CONCLUSION}

The $1-\mathrm{THz} 4^{\text {th }}$-harmonic gyrotron is theoretically studied. Due to strong ohmic loss at a high frequency, this paper proposes applying low-temperature cooling to increase the material conductivity and suppress the ohmic loss. A longer cavity with a higher $\mathrm{Q}$ factor is feasible operating in a low temperature condition, which will greatly enhance the interaction strength of the $4^{\text {th }}$ harmonic interaction. A preliminary design of $1-\mathrm{THz}, 4^{\text {th }}$-harmonic gyrotron using $T E_{4,8}^{(4)}$ mode and dynamic simulations have been carried out to analyze the mode competition between the operating modes and various competition modes. Due to the lower start oscillation current of $T E_{4,8}^{(4)}$ mode than the other modes, the $T E_{4,8}^{(4)}$ mode gradually becomes the dominant mode in the steady state regime. An output power of $1.15 \mathrm{~kW}$ excited by an $80-\mathrm{kV}$ 0.7-A axis encircling electron beam has been predicted.

\section{ACKNOWLEDGEMENTS}

Professor Chao-Hai Du would like to thank the Royal Society of London, UK, Newton Advanced Fellowships (NAF\R1\180121) for supporting this work.

\section{REFERENCE}

[1] N. C. Luhmann Jr, and W. A. Peebles, "Instrumentation for magnetically confined fusion plasma diagnostics," Rev. Sci. Instrum., vol. 55, no. 3, pp. 279-331, 1984.

[2] Y. Matsuki, K. Ueda, T. Idehara, R. Ikeda, K. Kosuga, I. Ogawa et al., "Application of Continuously Frequency-Tunable $0.4 \mathrm{THz}$ Gyrotron to Dynamic Nuclear Polarization for $600 \mathrm{MHz}$ Solid-State NMR," Journal of Infrared, Millimeter, and Terahertz Waves, vol. 33, no. 7, pp. 745-755, 2012.

[3] C.-H. Du, X.-B. Qi, L.-B. Kong, P.-K. Liu, Z.-D. Li, S.-X. $\mathrm{Xu}$ et al., "Broadband Tunable Pre-Bunched Electron Cyclotron Maser for Terahertz Application," IEEE Transactions on Terahertz Science and Technology, vol. 5, no. 2, pp. 236-243, 2015.

[4] K. R. Chu, "The electron cyclotron maser," (in English), Rev Mod Phys, vol. 76, no. 2, pp. 489-540, Apr 2004.

[5] T. Idehara, H. Tsuchiya, O. Watanabe, L. Agusu, and S. Mitsudo, "The first experiment of a THz gyrotron with a pulse magnet," Int J Infrared Milli, vol. 27, no. 3, pp. 319-331, 2006. 
[6] M. Y. Glyavin, A. G. Luchinin, and G. Y. Golubiatnikov, "Generation of $1.5-\mathrm{kW}, 1-\mathrm{THz}$ coherent radiation from a gyrotron with a pulsed magnetic field," Phys Rev Lett, vol. 100, no. 1, p. 015101, Jan 112008.

[7] S. Kao, C. Chiu, and K. Chu, "A study of sub-terahertz and terahertz gyrotron oscillators," Physics of Plasmas, vol. 19, no. 2, p. 023112, 2012.

[8] Chao-Hai Du, Shi Pan, Hui-Qi Bian, Pu-Kun Liu, "Time-domain multimode analysis of a $\mathrm{THz}$ gyro-TWT amplifier", IEEE Trans. Electron Devices, vol.65, no.4, pp:1550-1557, Apr. 2018. 